\title{
Generation of Low-Order Harmonics in Laser Ablation Plasmas
}

\author{
M. López-Arias ${ }^{\mathrm{a}, \mathrm{b}}$, M. Oujja ${ }^{\mathrm{a}}$, M. Sanz ${ }^{\mathrm{a}}$, R. de Nalda ${ }^{\mathrm{a}}$, R. A. Ganeev ${ }^{\mathrm{c}}$, M. Castillejo ${ }^{\mathrm{a},{ }^{*}}$ \\ ${ }^{a}$ Instituto de Química Física Rocasolano, CSIC, Serrano 119, 28006 Madrid, Spain. \\ ${ }^{b}$ Unidad Asociada Departamento de Química Física I, Facultad de Ciencias Químicas, \\ Universidad Complutense de Madrid, 28040 Madrid, and Instituto de Estructura de la \\ Materia, CSIC, Serrano 123, 28006 Madrid, Spain \\ ${ }^{c}$ Institute of Electronics, Uzbekistan Academy of Sciences, Akademgorodok, 33, Dormon \\ Yoli Street, Tashkent 100125, Uzbekistan.
}

\begin{abstract}
Third and fifth harmonics of a pulsed infrared laser $(1064 \mathrm{~nm})$ delivering pulses of nanosecond duration have been generated in the laser ablation plasmas of various materials including the metals $\mathrm{Al}$ and $\mathrm{Fe}$ and the highly ionic insulators $\mathrm{MgF}_{2}$ and $\mathrm{NaCl}$. The harmonics were generated in a process triggered by laser ablation followed by frequencyup-conversion of the fundamental laser beam that propagates parallel to the target surface. The spatial and temporal behaviour of the harmonics reveals the distinctive composition and dynamics of each plasma, gives insight on the species that act as nonlinear media and allows the comparison of different processes that control the generation efficiency. Loworder harmonic generation thus emerges as a powerful and universal technique for monitoring and diagnostics of ablation plasmas while, at the same time, the results presented serve to guide the choice of solid materials for efficient high harmonic generation using ultrashort pulses in the femtosecond regime.
\end{abstract}

Keywords: Laser ablation, Ablation Plasma, Harmonic Generation

\footnotetext{
*Corresponding author, E mail: marta.castillejo@iqfr.csic.es
} 


\section{Introduction}

Year 2011 marks the 50th birthday of Nonlinear Optics [1]. The perturbative treatment of Bloembergen [2] served to introduce phase-matching techniques that allowed efficient frequency up-conversion of an initial fundamental frequency. In this perturbative regime, which holds when using focused common nanosecond (ns) lasers, three and four wave mixing processes account for frequency doubling and tripling in the degenerate case, where all fundamental frequencies involved are equal. Techniques based in low-order harmonic generation (HG), using ns laser pulses duration, have made possible for decades the generation of coherent light in the ultraviolet and vacuum ultraviolet spectral regions $[3,4]$ to be used in spectroscopy and molecular photodissociation studies $[5,6]$. On the other hand, high-order harmonic generation (HHG) [7] requires driving laser pulses of higher intensities $\left(10^{14}-10^{15} \mathrm{~W} / \mathrm{cm}^{2}\right)$ typically achieved with femtosecond (fs) pulses. In a gas medium, and due to basic symmetry considerations, only odd harmonics are generated. Atomic and molecular gases and vapours in static cells and in gas jets [8,9] are widely used as nonlinear media for $\mathrm{HG}$.

Pulsed laser ablation of a solid target allows the entraining of a large variety of species in a gas flow, including atoms and molecules (both neutral and ionized), highly charged particles, clusters and aggregates. This occurs through the violent evaporation from the solid and subsequent plasma dynamics, which involves aggregation, fragmentation and electron impact ionization. The nonlinear optical properties of such species, or their behaviour under strong laser irradiation, can thus be studied through this technique $[10,11]$. This makes possible to extend the techniques of HG and HHG to some nonlinear species 
based in polar molecules, atomic ions or nanoparticles, which are not readily available in the gas phase [12-16].

Recently, we have undertaken the study of low-order HG in ablation plumes of dielectric, semiconductor and metal solid targets using Q-switched Nd:YAG lasers with pulses of ns duration [14-16]. These studies have lead us to propose HG, performed in configurations that allow spatiotemporal exploration of different regions of the plasma, as an advantageous tool for plasma diagnosis due to its high versatility and absence of limitations regarding mass range, spectral range, etc. On the other hand we aim at understanding the impeding and restricting processes of $\mathrm{HG}$ in laser generated plasmas and identifying the media that lead to highly efficient up-conversion, as the analysis of low-order harmonics allows definition of the processes which hamper further enhancement of conversion efficiency in HHG processes.

In this paper we report on low-order $\left(3^{\text {rd }}\right.$ and $\left.5^{\text {th }}\right)$ HG in species present in ablation plasmas of the metals $\mathrm{Al}$ and $\mathrm{Fe}$ and on the dielectrics $\mathrm{MgF}_{2}$ and $\mathrm{NaCl}$. The harmonic signal distribution resolved in the spatiotemporal dimensions reveals distinctive characteristics related to the composition and dynamics of the ablation plume of each material at the selected ablation wavelength. We believe the interest of this work is twofold: on one hand, it confirms the potential of the low-harmonic generation for plasma diagnostics of all types of target materials; on the other, it provides a guide for the selection of ablation targets for efficient generation of short wavelength coherent light by high harmonic up-conversion of ultraintense fs pulses.

\section{Experimental}


The experimental set up has been described earlier [14,15] and a brief account is given here. Ablation plasmas of the different solid targets were generated in vacuum through normal incidence irradiation with the fundamental, second or fourth harmonic of a Qswitched Nd:YAG laser (Quantel, Brilliant B, 6 ns full-width-half-maximum, FWHM, $1064 \mathrm{~nm}, 532$ and $266 \mathrm{~nm}$, respectively) that was operated at $10 \mathrm{~Hz}$ using pulse energies of 2-35 mJ. This ablation beam was focused with a $17 \mathrm{~cm}$ focal length lens to a spot of 0.4 $\mathrm{mm}$ diameter on the surface of the target, generating maximum intensities of $\sim 4.5 \times 10^{9}$ $\mathrm{W} / \mathrm{cm}^{2}$, above the ablation thresholds of the different materials. The vacuum chamber was kept at $1 \mathrm{~Pa}$ background pressure, and the samples were mounted on a rotating holder to avoid cratering. The harmonic driving IR fundamental radiation was delivered by a second Nd:YAG laser (Lotis TII LS-2147, 15 ns FWHM, 1064 nm, $10 \mathrm{~Hz}$ ) propagating parallel to the target surface in such a way that it intersected the ablation plasma at a controllable distance from the surface (distances of 0.6 and $1 \mathrm{~mm}$ were employed). Typical pulse energies were in the range of 20-650 mJ. The beam was focused with a $20 \mathrm{~cm}$ focal length lens to a spot of around $0.04 \mathrm{~mm}$ diameter, so that laser intensities in the harmonic generating region reached values of $\sim 1.0 \times 10^{11}-3.5 \times 10^{12} \mathrm{~W} / \mathrm{cm}^{2}$. The delay between this driving pulse and the ablation pulse was controlled electronically in the range from 0 to 10 $\mu \mathrm{s}$.

Low-order harmonics of the driving laser were produced in the interaction region, and those propagated collinearly with the driving beam. Two highly IR transmissive, UV reflective mirrors were placed at the exit of the vacuum chamber to avoid damage of the detector with the intense IR beam. After the mirrors, a system consisting on a monochromator (TMc300 Bentham) with a ruled 300 lines/mm grating coupled to a time-gated, intensified charge- 
coupled-device (ICCD, 2151 Andor Technologies) was used for detection of the generated harmonics. The temporal resolution of the acquisition system, taking into account the jitter and the minimum gate width, is 10 ns. For measurements, third (TH) and fifth (FH) harmonics were detected in the same spectral window by monitoring, together with the TH at $355 \mathrm{~nm}$, the second order of the $\mathrm{FH}$ at $425.6 \mathrm{~nm}(2 \mathrm{FH})$. Electronically excited species generated in the plasma produced optical emissions that could, under some conditions, be detected simultaneously with the harmonics. Typical acquisitions corresponded to an accumulation of 150 laser shots and a detection gate of 100 ns. These settings favour the discrimination of harmonic signals from the temporally broad spontaneous emissions of the ablation plasma.

The metal targets employed in the experiments were purchased from Kurt Lesker (Al, $99.99 \%$ ) and GoodFellow (Fe, $99.5 \%$ ). For experiments with $\mathrm{MgF}_{2}$ and $\mathrm{NaCl}$, optically flat, $5 \mathrm{~mm}$ thick windows were used as targets.

\section{Results and discussion}

The ablation wavelength selected for harmonic generation studies herein was chosen for each material to ensure the survival of the target by repetitive laser irradiation and at the same time to produce an intense luminous ablation plume. According to these criteria the metals were ablated at $1064 \mathrm{~nm}$, while the second and fourth harmonics at 532 and $266 \mathrm{~nm}$ were selected for the dielectrics $\mathrm{MgF}_{2}$ and $\mathrm{NaCl}$ respectively. Spectra were acquired in the region of interest for a given distance of the driving laser from the target surface and for a range of temporal delays between the ablation and driving lasers. In those measurements, signals from the harmonics of the IR driving laser were recorded simultaneously with laserinduced spontaneous emissions of excited species in the plume. Figure 1 displays 
illustrative examples of the emission spectra of the $1064 \mathrm{~nm}$ ablation plumes of $\mathrm{Al}, \mathrm{Fe}$ and of the emission spectrum of the $266 \mathrm{~nm}$ ablation plume of $\mathrm{NaCl}$. Those were recorded in the presence of the IR driving laser propagating at a distance of $0.6 \mathrm{~mm}$ from the surface. The sharp feature at $355 \mathrm{~nm}$ is assigned to the $\mathrm{TH}$ and that at $425.6 \mathrm{~nm}$ to the $\mathrm{FH}$ detected in second order of the grating (2FH). Spontaneous emissions (also observed in absence of the driving laser) in $\mathrm{Al}, \mathrm{Fe}$ and $\mathrm{NaCl}$ plumes are respectively attributed to electronically excited atomic $\mathrm{Al}, \mathrm{Fe}$ and $\mathrm{Na}$ (neutrals or ions). The second orders of some atomic emissions are also detected above $400 \mathrm{~nm}$. Assignments are shown in the Figure. For $\mathrm{MgF}_{2}$ (not shown), the $\mathrm{MgF}\left(\mathrm{A}^{2} \Pi-\mathrm{X}^{2} \Sigma^{+}\right)$molecular emission at around $360 \mathrm{~nm}$ [17] is observed together with emissions assigned to $\mathrm{Mg}$ neutrals and ions.

The nature of the emissions detected at 355 and $425.6 \mathrm{~nm}$ was further confirmed by measuring the power dependence with respect to the energy of the driving beam. Figure 2 shows the measurements for the 1064-nm laser-generated plasma of Al. The slopes obtained in log-log plots were compatible with the value of 3 and 5 , expected for the behaviour of the third and fifth harmonics, respectively, in the perturbative regime. These dependences were observed for $\mathrm{TH}$ and $2 \mathrm{FH}$ generated in the plumes of all the materials considered here. It should be noted that the relative intensity of the $\mathrm{TH}$ and $2 \mathrm{FH}$ signals does not directly reflect that of the generated third and fifth harmonics, on one hand because the latter was measured by the signal in second order, and on the other because the spectra were not corrected by the response of the detection system. However, the high intensity of the signal corresponding to the $\mathrm{FH}$ in the case of $\mathrm{Al}$, as compared with that observed in the plume of $\mathrm{Fe}$ or of the dielectrics $\mathrm{MgF}_{2}$ and $\mathrm{NaCl}$, suggests that in the $\mathrm{Al}$ ablation plume, FH generation could possibly be selectively favoured by a resonant effect 
due to the spectral proximity of a high oscillator strength transition. In this metal, atomic transitions termed ${ }^{2} \mathrm{P}^{\circ}-{ }^{2} \mathrm{~S}$ (for neutral $\mathrm{Al}$ ) and ${ }^{1} \mathrm{~S}-{ }^{3} \mathrm{P}^{\circ}$ (for singly ionized $\mathrm{Al}$ ) [18] are resonant with the energy of four photons of the $1064 \mathrm{~nm}$ driving laser. However, since the initial and final states in both transitions have opposite parity, they could not couple by a four-photon transition. Lower oscillator strength resonances to five photon transitions of the right parity (involving the $3 \mathrm{p}^{2} \mathrm{~ns}$ Rydberg series) could possibly contribute to enhancement of the FH signal although in this case resonant absorption of the newly generated frequency would result in a decreased efficiency yield. On the other hand the effect of transient resonances should not be disregarded, since the fairly high intensity of the driving laser will induce an AC Stark shift of the states. This shift will especially affect the more loosely bound Rydberg states, which roughly will be shifted by the ponderomotive potential and can actually also influence phase matching. Despite the several contributions, as mentioned, it can be concluded at this point, that the observed relatively high $\mathrm{FH}$ yield of the Al plume could be due to the interplay of the mentioned effects.

In all studied targets, the onset of harmonic signal coincided with the appearance of a luminous plume induced by the ablation laser and the HG efficiency was observed to depend on the ablation pulse energy. Figure 3 shows the case of $\mathrm{TH}$ and $2 \mathrm{FH}$ signals generated in the plumes of $\mathrm{Al}$ and Fe. Harmonic signals rise with ablation pulse energy until a maximum value is reached at 7 and $15 \mathrm{~mJ}$ for $\mathrm{Al}$ and $\mathrm{Fe}$ respectively. This difference is ascribed to the higher ablation fluence threshold for Fe which is twice as large as for Al. The increase of harmonic signal with ablation pulse energy is due to the higher amount of material ejected from the target and correspondingly to the growing local density 
of nonlinear emitters (through the expected quadratic dependence with local density [19]). The signal decline at higher ablation pulse energy should be attributed to the enhanced free electron density in the plasma, which gives a detrimental contribution to the phase mismatch [11]. However, at the higher pulse energies, the possibility of changed plume composition, in terms of mass distribution and degree of ionization, cannot be ruled out. The FH in Al decays faster with ablation energy than the TH, Figure 3(a), while for Fe, Figure 3(b), it is optimized at somewhat lower ablation energy. These differences are related to the different composition and dynamics of the plumes of these two metals and possibly, in the case of Fe, to the dissimilar density and phase mismatch conditions of the nonlinear species contributing to the generation of each harmonic. The different sensitivity to phase mismatch of third and fifth harmonics could partly explain their different behaviour with ablation pulse energy.

The behaviour of the emitted harmonics was studied as a function of the spatiotemporal region of the plume sampled by the driving laser. First we monitored the dependence on the delay between ablation and driving pulses and second with the distance of the propagation direction of the driving laser to the target surface ( $x$ coordinate). In each case, the ablation laser energy was set to the value of maximum $\mathrm{TH}$ generation efficiency. Changes in this value did not affect qualitatively the observed general features.

Figure 4(a-c) shows the emitted $\mathrm{TH}$ and $\mathrm{FH}$ intensities in the plumes of $\mathrm{Al}, \mathrm{Fe}$ and $\mathrm{MgF}_{2}$ as a function of the time delay between ablation and driving lasers. The ablation laser energy chosen for these measurements corresponds to around $1.5-2$ times the ablation threshold for each material. The curves obtained were normalized for easier comparison. A single temporal component is observed for all targets, although differences in the width of the 
temporal delay maximum may be indicative of different kinetic energies or masses of the ejected nonlinear emitting species. The peak up-conversion yield should be obtained when the combined contribution of density of nonlinear emitters and phase mismatch conditions is highest, taking into account that optimum phase-matching conditions are achieved when the free-electron-induced dispersion is overcome by dispersion attributable to atoms, ions and other nonlinear species created in the ablation plume. For the metals, the delay at which the $\mathrm{TH}$ reaches its maximum intensity scales with the atomic number. This can be explained as ejection of lighter atoms and ions from the target surface, presumably responsible for the earlier stages of $\mathrm{HG}$ [15], reach the region sampled by the fundamental beam earlier as compared with heavier species. It is also interesting to note that the relative strength of the $\mathrm{TH}$ and $\mathrm{FH}$ is independent of the time delay for $\mathrm{Al}$ but not for $\mathrm{Fe}$. In the former this indicates that this ratio is not governed by the instantaneous density of nonlinear plume species [20] while for the latter, the earlier rise and decay of the FH signal could indicate that different nonlinear species, ejected at different velocities from the target, are responsible for the generation of third and fifth harmonics (as already indicated above). Comparison of the optimum delay values obtained for the metal plumes with those of $\mathrm{MgF}_{2}$ or $\mathrm{NaCl}$ is not possible, as these were not measured using the same ablation wavelength, a parameter that determines the ablation mechanism and therefore has a strong influence on the velocity of the ejected species [21-23].

The measurements of the intensity of the harmonic signals as a function of the distance to the target $x$ give information of the spatial dimensions of the laser-created nonlinear media. The behaviour of the $\mathrm{TH}$ and $\mathrm{FH}$ generated by the IR laser plume of the metals $\mathrm{Al}$ and $\mathrm{Fe}$ is plotted in Figure 5. For each distance probed, the delay between the ablation and the 
fundamental beams was set at the value corresponding to the optimum signal. As observed, the harmonic yield is negligible at $2.5 \mathrm{~mm}$ away from the target. Assuming optimum phase matching conditions, the dependence of harmonic signal with the distance to the target $x$ is mediated by the corresponding dependence of the product $d^{2} L_{p}{ }^{2}$, where $d$ is the density and $L_{p}$ is the length of nonlinear medium $[15,16]$. In an isotropic emission of nonlinear species from the target, $d$ scales with $x^{-2}$. As $L_{p}$ is expected to be proportional to $x$, the harmonic signal should be compatible with a $x^{-2}$ dependence. As showed in Figure 5, harmonic signals closely follow this dependence.

Finally we obtained an estimate of the harmonic generation efficiency in the plumes of the investigated targets [14]. For determination of the TH generation efficiency, a strongly attenuated $\mathrm{TH}$ of another Nd:YAG laser $(355 \mathrm{~nm})$, was made to propagate along the direction of propagation of the driving beam with similar propagation geometry and identical detection conditions to the $\mathrm{TH}$ emitted from the ablation plume of a given target. Attenuation was tuned so that the signal magnitude was comparable to that obtained from the $\mathrm{TH}$ generated in the plume. Measurement of the pulse energy prior to attenuation, together with a well characterized attenuation factor, allowed us to estimate pulse energies, which provided an absolute reference for the energy of the $\mathrm{TH}$ emitted from the plume. Errors due to uncertainties in the divergence of the beams were minimized by using the widest slits possible in the monochromator and checking that no significant spatial selection was taking place. This procedure yielded an efficiency value of $\approx 4 \times 10^{-5}$ for the TH generated in the $1064 \mathrm{~nm}$ plumes of $\mathrm{Al}, \mathrm{Fe}$ and in the $532 \mathrm{~nm}$ plume of $\mathrm{MgF}_{2}$. The highest TH generation efficiency was obtained for the $266 \mathrm{~nm}$ plume of $\mathrm{NaCl}$, with an estimated value of $4 \times 10^{-4}$. This high efficiency may be related with the highest density of 
nonlinear scatterers produced under the ablation conditions selected for this material and their correspondingly higher nonlinear susceptibility. Work is in progress to further clarify the different low-order harmonic generation efficiencies observed in the various materials of this study, as this constitutes a highly relevant issue for the selection of ns ablation plumes to be used as nonlinear media for HHG.

\section{Conclusions}

Low-order harmonic generation of a $1064 \mathrm{~nm}$ laser has been investigated in laser ablation plasmas of the metals aluminium and iron and of the dielectrics $\mathrm{MgF}_{2}$ and $\mathrm{NaCl}$. The spatiotemporal structure of the third and fifth harmonics has been determined through the use of an adequate geometry and a two-laser pulse sequence that permits the exploration of compositionally distinct regions of the plasma in terms of kinetic energy or in terms of species ejected from the solid target. The obtained distributions depend on the target

material, on the wavelength of the laser used for ablation and on the presence of resonant transitions of the plasma species. The ablation plasma with highest third harmonic conversion efficiency corresponds to the $266 \mathrm{~nm}$ plume of the ionic insulator $\mathrm{NaCl}$, with an estimated value of $4 \times 10^{-4}$. Work is in progress to explore the potential of the ns ablation plasmas studied herein for the generation of high-order harmonics of fs driving lasers.

\section{Acknowledgements}

Funding has been provided by Ministry of Science and Innovation of Spain (MICINN) under Project CTQ2010-15680. M.O. and M.S. acknowledge CONSOLIDER CSD200700058 and CAM (Geomateriales P2009/MAT 1629) respectively for contracts. M. L.-A. thanks CSIC for a JAE-Pre contract. 


\section{References}

[1] P.A. Franken, A.E. Hill, C.W. Peters, and G. Weinreich, Phys. Rev. Lett. 7, 118 (1961).

[2] J.A. Armstrong, N. Bloembergen, J. Ducuing, and P.S. Pershan, Phys. Rev. 127, 1918 (1962).

[3] R. Hilbig and R. Wallenstein, IEEE J. Quantum Elec. 17, 1566 (1981).

[4] M. Castillejo, J.Y. Zhou, and M.H.R. Hutchinson, Appl. Phys. B 45, 293 (1988).

[5] M. Castillejo, M. Martin, R. de Nalda, and M. Oujja, Chem. Phys. Lett. 237, 367 (1995).

[6] A.H. Zanganeh, J. H. Fillion, J. Ruiz, M. Castillejo, J. L. Lemaire, N. Shafizadeh, and F. Rostas, J. Chem. Phys. 112, 5660 (2000).

[7] X.F. Li, A. L'Huillier, M. Ferray, L.A. Lompre, and G. Mainfray, Phys. Rev. A 39, 5751 (1989).

[8] M. Lein, R. De Nalda, E. Heesel, N. Hay, E. Springate, R. Velotta, M. Castillejo, P.L. Knight, and J.P. Marangos, J. Mod. Optic. 52, 465 (2005).

[9] C. Lyngå, A. L’Huillier, and C.-G. Wahlström, J. Phys. B: At. Mol. Opt. Phys. 29, 3293 (1996).

[10] R.A. Ganeev, V.I. Redkorechev, and T. Usmanov, Opt. Commun. 135, 251 (1997).

[11] R.A. Ganeev, J. Phys. B: At. Mol. Opt. Phys. 40, R213 (2007).

[12] B.S. Zhao, M. Castillejo, D.S. Chung, B. Friedrich, and D. Herschbach, Rev. Sci. Instrum. 75, 146 (2004). 
[13] N. Hay, R. de Nalda, T. Halfmann, K.J. Mendham, M.B. Mason, M. Castillejo, and J.P. Marangos, Eur. Phys. J. D, 14, 231 (2001).

[14] M. Oujja, R. de Nalda, M. López-Arias, R. Torres, J.P. Marangos, and M. Castillejo, Phys. Rev. A 81, 043841 (2010).

[15] R. de Nalda, M. López-Arias, M. Sanz, M. Oujja, and M. Castillejo, Phys. Chem. Chem. Phys. 13, 10755 (2011).

[16] M. López-Arias, M. Oujja, M. Sanz, R.A. Ganeev, G. S. Boltaev, N. Kh. Satlikov, R. I. Tugushev, T. Usmanov, and M. Castillejo, submitted.

[17] K. P. Huber and G. Herzberg, Molecular Spectra and Molecular Structure. Constants of Diatomic Molecules (van Nostrand Reinhold, New York, 1979).

[18] National Institute of Standards and Technology, Atomic Spectra Database, $<$ http://physics.nist.org>.

[19] J.F. Reintjes, Nonlinear Optical Parametric Processes in Liquids and Gases, (Academic Press, Orlando, 1984).

[20] W. Theobald, C. Wülker, F.P. Schäfer, and B.N. Chichkov, Optics Comm. 120, 177 (1995).

[21] M. Sanz, M. Walczak, M. Oujja, A. Cuesta, and M. Castillejo, Thin Solid Films 517, 6546 (2009).

[22] M. Sanz, M. Walczak, R. de Nalda, M. Oujja, J.F. Marco, J. Rodríguez, J.G. Izquierdo, L. Bañares, and M. Castillejo, Appl. Surf. Sci. 255, 5206 (2009). 
[23] M. Sanz, R. de Nalda, J.F. Marco, J.G. Izquierdo, L. Bañares, and M. Castillejo, J. Phys. Chem. C 114, 4864 (2010). 


\section{Figure captions}

Figure 1. Spectra of emissions detected upon ablation and in the presence of the fundamental $1064 \mathrm{~nm}$ driving beam of $\mathrm{Al}(\mathrm{a}), \mathrm{Fe}(\mathrm{b})$ and $\mathrm{NaCl}$ (c) targets. The conditions are as follows. For $\mathrm{Al}$ and Fe the ablation wavelength was $1064 \mathrm{~nm}$ and the energy per pulse $7 \mathrm{~mJ}$ and $12 \mathrm{~mJ}$ respectively; the driving beam with pulse energy of $560 \mathrm{~mJ}$ was propagating at $0.6 \mathrm{~mm}$ from the target with a delay of $200 \mathrm{~ns}$. For $\mathrm{NaCl}$ the ablation wavelength was $266 \mathrm{~nm}$ and the energy per pulse $10 \mathrm{~mJ}$; the driving beam, with pulse energy of $425 \mathrm{~mJ}$, was propagating at $1.0 \mathrm{~mm}$ from the target with a delay of $450 \mathrm{~ns}$. The lines corresponding to third harmonic $(\mathrm{TH})$ and the second order of the fifth harmonic $(2 \mathrm{FH})$ and of the atomic lines are indicated.

Figure 2. Log-log plot of the signals corresponding to the $\mathrm{TH}$ (open circles) and of the second order of the $\mathrm{FH}(2 \mathrm{FH}$, solid circles) of the driving laser generated in the $1064 \mathrm{~nm}$ ablation plume of $\mathrm{Al}$ as function of the laser pulse energy. The signals, detected at a distance of $0.6 \mathrm{~mm}$ from the target, were obtained using an ablation pulse energy of $7 \mathrm{~mJ}$, with a delay of $150 \mathrm{~ns}$. As indicated, the slopes are compatible with a third and fifth order non-linear processes. The continuous lines are the best fits to the data.

Figure 3. Normalized intensity of the TH (open circles) and FH (solid circles) of the driving laser as function of the ablation pulse energy for (a) $\mathrm{Al}$ and (b) Fe. Results were obtained with driving laser pulse energy of $560 \mathrm{~mJ}$, distance to target $0.6 \mathrm{~mm}$ and delay 200 ns. The continuous lines are visual guides.

Figure 4. Normalized harmonic signals of the third (open circles) and fifth (solid circles) orders of the IR driving laser in ablation plasmas as a function of the delay between the 
ablation event and the arrival of the driving laser, and at a distance of $0.6 \mathrm{~mm}$ from the surface of the target of: (a) Al induced by a $1064 \mathrm{~nm}$ ablation laser with pulse energy of 7 $\mathrm{mJ}$; (b) Fe induced by a $1064 \mathrm{~nm}$ ablation laser with pulse energy of $14 \mathrm{~mJ}$; (c) $\mathrm{MgF}_{2}$ induced by a $532 \mathrm{~nm}$ ablation laser with pulse energy of $10 \mathrm{~mJ}$. The driving laser power was $560 \mathrm{~mJ}$ for $\mathrm{Al}$ and $\mathrm{Fe}$ and $425 \mathrm{~mJ}$ for $\mathrm{MgF}$. The continuous lines are visual guides.

Figure 5. Normalized intensity of the $\mathrm{TH}$ and $2 \mathrm{FH}$ signals generated in the $1064 \mathrm{~nm}$ ablation plumes of: (a) $\mathrm{Al}$ and (b) $\mathrm{Fe} \mathrm{nm}$ as the position of the fundamental IR beam is displaced from the surface of the metal target ( $x$ distance). For each distance probed, the delay between the ablation beam and the fundamental beam was set at the value corresponding to the optimum signal. The solid lines represent a $x^{-2}$ decay. 


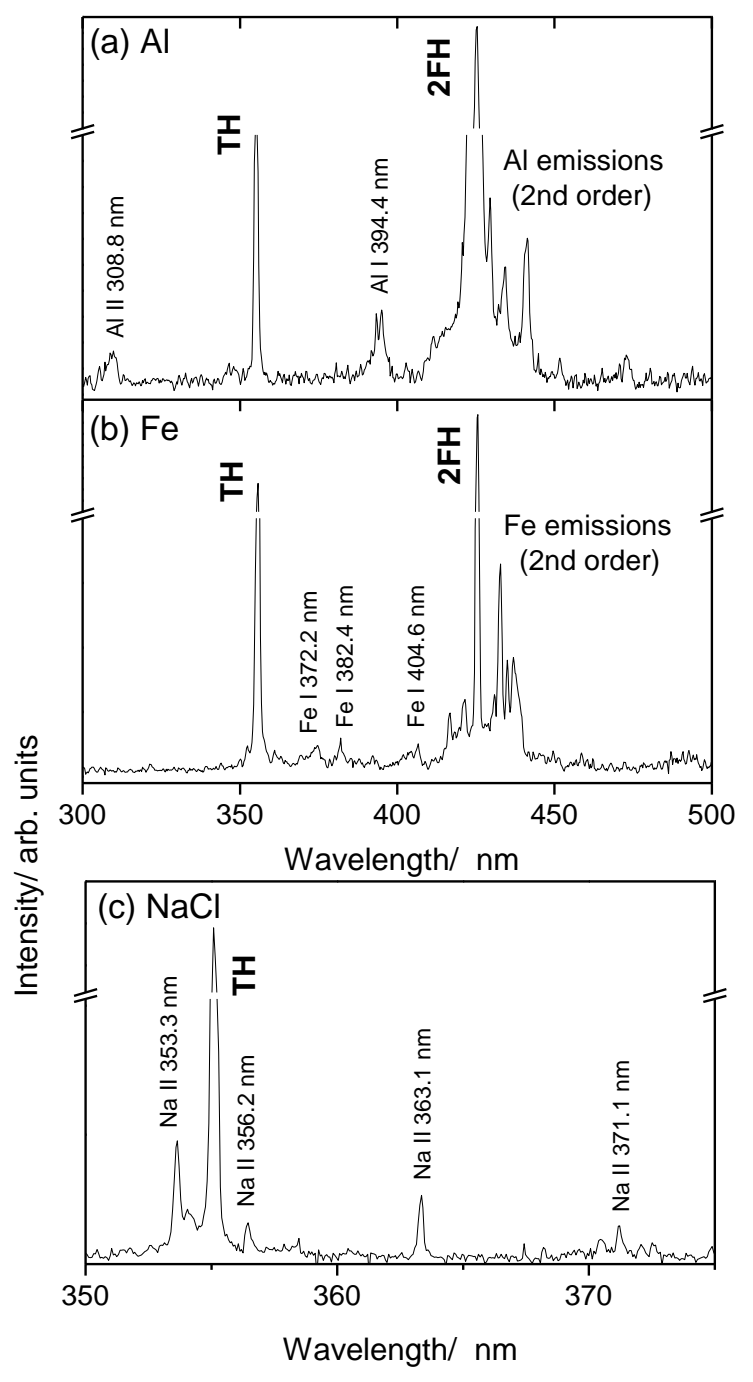

Figure 1. 


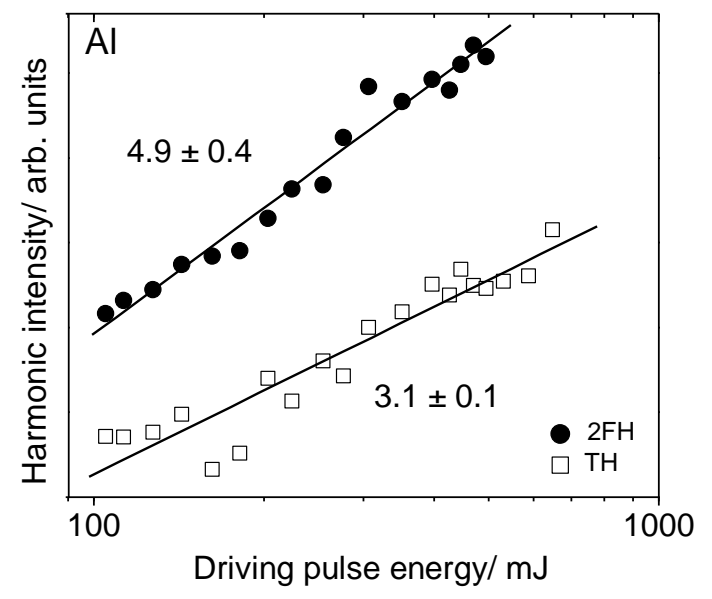

Figure 2 


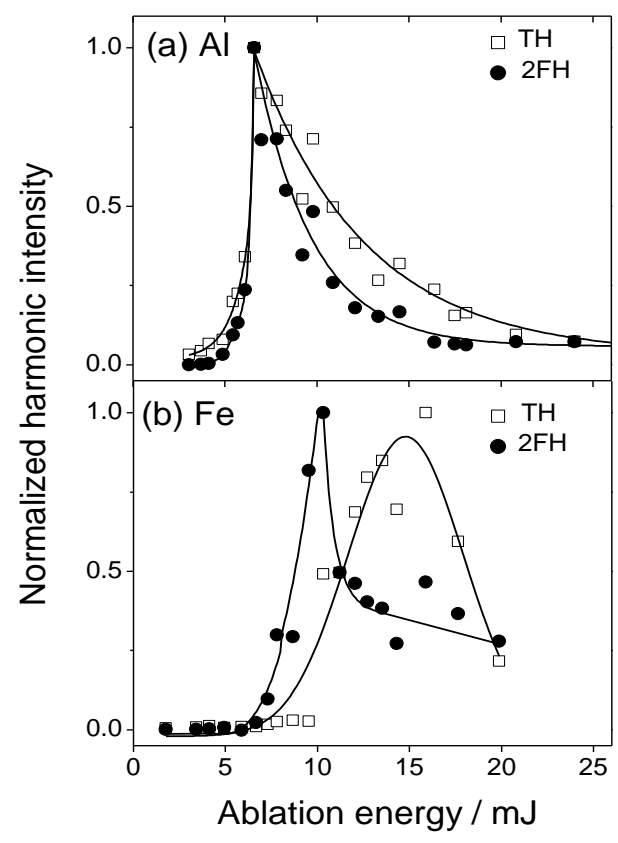

Figure 3 

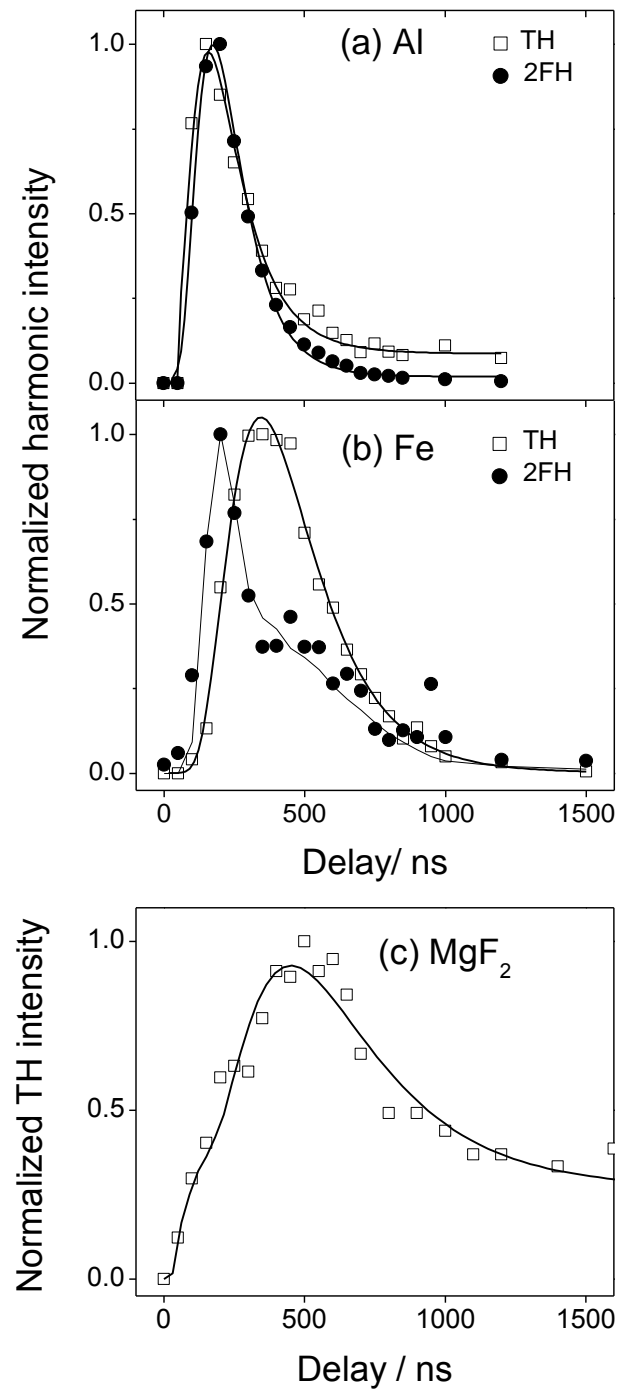

Figure 4 


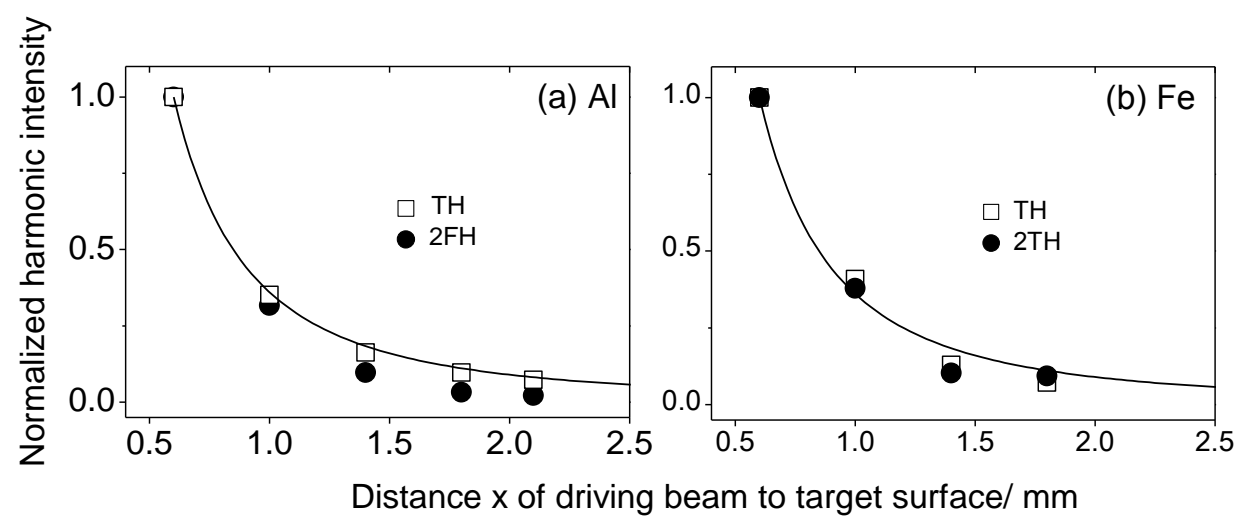

Figure 5 